\title{
Nachruf: Univ. Prof. Dr. Franz Traxler
}

Die österreichische Soziologie hat einen ihrer international renommiertesten Vertreter verloren: Franz Traxler ist im Jänner 2010 völlig überraschend gestorben. Er stand mit 58 Jahren mitten in einem sehr aktiven Leben, das er zum Großteil der Wissenschaft gewidmet hatte. Ich lernte Franz Traxler auf der Wirtschaftsuniversität kennen, als ich im Jahr 1984 als Studierendenvertreter Mitglied seiner Habilitationskommission war. Zuletzt traf ich ihn zwei Wochen vor seinem Tod anlässlich eines Rigorosums, das wir gemeinsam abnahmen. In der langen Zeit dazwischen gab es viele Anlässe zu Begegnungen und Kooperationen, bei denen ich ihn immer als genauen, verlässlichen und unterstützenden Hochschullehrer, Wissenschafter und Kollegen erlebte.

Seine wissenschaftliche Laufbahn begann er als Universitätsassistent am Institut für Allgemeine Soziologie und Wirtschaftssoziologie an der Wirtschaftsuniversität Wien, an der er studiert hatte. Dort habilitierte er sich im Alter von 34 Jahren und war anschließend als Abteilungsleiter an der Verwaltungsakademie des Bundes in Wien tätig. 1992 wurde er als ordentlicher Universitätsprofessor an die Universität Wien berufen. Seine Arbeitsgebiete reichten von der Industrie- und Organisationssoziologie und der Politischen Soziologie bis zu den Arbeitsbeziehungen und insbesondere der vergleichenden Verbändeforschung sowie dem Theorievergleich auf der Grundlage empirischer Forschung im Bereich der Wirtschafts- und Organisationssoziologie. In zahlreichen Publikationen in renommierten Zeitschriften und Verlagen gab er der internationalen Industrial-Relations-Forschung und insbesondere der vergleichenden Analyse der Institutionen der Arbeitsbeziehungen entscheidende Impulse.

Über seine Forschungs- und Lehrtätigkeit hinaus trug er in verschiedenen Funktionen zur Stärkung der österreichischen Soziologie und der internationalen Industrial-Relations-Forschung bei. So war er von 1993 bis 1997 Präsident der Österreichischen Gesellschaft für Soziologie und koordinierte ab 1996 das Research Network on Industrial Relations, Labour Market Institutions and Employment der European Sociological Association. Darüber hinaus war er als Konsulent einer Reihe internationaler Organisationen tätig: der Europäischen Stiftung zur Verbesserung der Lebens- und Arbeitsbedingungen, der Europäischen Kommission, der OECD und der ILO. Die umfassenden Forschungsarbeiten, die sich zuletzt auf die Wirkungen der Institutionen der Arbeitsbeziehungen und des Arbeitsmarktes im internationalen Vergleich konzentrierten, ebenso wie die internationalen Aktivitäten und Kontakte lassen erkennen, wie groß die Lücke ist, die sein Tod in der österreichischen Wissenschaft hinterlässt.

Auch wenn seine Produktivität und seine Erfolge anderes vermuten lassen könnten, hatte Franz Traxler auch ein reiches Leben außerhalb des Berufs. Erwähnt sei nur die Betreuung seiner nun sechsjährigen Tochter. Auch in dieser Hinsicht stand er mitten im Leben, das viel zu früh endete. 